\title{
FREQÜÊNCIA DE LESÕES PERINEAIS OCORRIDAS NOS PARTOS VAGINAIS EM UMA INSTITUIÇÃO HOSPITALAR
}

\author{
Frequency of perineal lesions occurred during natural child \\ labour in a hospital institution.
}

\section{Frecuencia de lesiones perineales ocurridos en los partos naturales en una institución hospitalaria.}

Jaqueline de Oliveira Santos

Lidiane Coleoni ${ }^{4}$
Izabel Cristina Bolanho

Márcia Alessandra de Oliveira ${ }^{5}$
Jaqueline Queiroz Carlos da Mota ${ }^{3}$

\section{RESUMO}

Durante o parto normal, a maioria das mulheres sofre algum tipo de lesão perineal, em razão de lacerações espontâneas ou como conseqüência da incisão cirúrgica - episiotomia. Este estudo, do tipo descritivo, com abordagem quantitativa, visa levantar a freqüência de lesões perineais ocorridas em mulheres durante o parto vaginal em uma instituição hospitalar que exerce a obstetrícia tradicional. A amostra foi composta por 279 prontuários de mulheres que pariram na instituição durante o período de julho a dezembro de 2006. Em 11,82\% dos prontuários não foi relatada a ocorrência de lesões. A episiotomia foi realizada em $86,99 \%$ das mulheres, $3,25 \%$ sofreram episiotomia e lacerações perineais de $1^{\circ}$ e $2^{\circ}$ graus, $9,76 \%$ tiveram o períneo íntegro. A prática da episiotomia não foi justificada nos prontuários, sua realização ocorreu sem qualquer enfoque seletivo, sendo praticada rotineiramente na instituição, demonstrando que ainda persiste o desrespeito aos direitos humanos adquiridos pelas mulheres.

Palavras-chave: Parto Natural. Períneo. Episiotomia. Saúde da Mulher.

\begin{abstract}
During the process of natural child labour, most of women suffers some kind of perineal trauma when they go into natural childbirth labor, due to spontaneous perineum lacerations or episiotomies. The objective of this study of quantitative and descriptive approach is to document the number of perineal traumas occurred in women during natural childbirths in a hospital institution. This study was created based on 279 files of women who delivered their babies by natural birth a hospital institution that practiced tradicional obstetricsduring a period from July to December 2006. In 11,82\% of the cases no lesions were reported. The episiotomy was practiced in $86,99 \%$ of the women and $3,25 \%$ suffered episiotomy and perineal lacerations in 1 and 2 degrees, $9,76 \%$ of the women had their perineum intact. The practice of the episiotomy was not justified in the files and it is practiced as a routine without any selective criteria by the institution, demonstrating that the disrespect to human rights of women still persists.
\end{abstract}

Keywords: Natural Childbirth. Perineum. Episiotomy. Women's health.

\begin{abstract}
Resumen
Durante el proceso de parto normal, la mayoría de las mujeres sufre algún tipo de trauma del períneo, debido a laceraciones espontáneas del períneo o por la incisión quirúrgica - las episiotomías. Este estudio de tipo descriptivo con un abordaje cuantitativo y intenta registrar el número y frecuencia de de traumas perineales ocurridos en mujeres durante el parto normal en una instituición hospitalar que practica obstetricia tradicional. La muestra fue recogida de 279 casos clínicos de mujeres que tuvieron partos normales en la institución, durante el período de julio a diciembre de 2006. En 11,82\% de los casos no hubo relatos de. La episiotomía fue practicada en $86.99 \%$ de las mujeres, $3.25 \%$ sufrieron de episiotomía y de laceraciones de primero y segundo grados, $9.76 \%$ tuvieron el períneo intacto. Por lo tanto, concluímos que la episiotomía es practicada rutinariamente sin justificación y sin enfoque selectivo y en la instituición. Esto demuestra que persiste una clara falta de respeto a los derechos humanos de las mujeres.
\end{abstract}

Palabras-claves: Parto normal Perineo. Episiotomía. Salud de la mujer 


\section{INTRODUCÃO}

Impulsionado pelo movimento em prol da humanização do nascimento e do parto e com base nas evidências científicas, a Organização Mundial de Saúde (OMS), publicou em 1996, um guia prático para a assistência ao parto normal, no qual preconizou o respeito ao processo fisiológico e a dinâmica de cada nascimento 1 .

Seguindo as mesmas diretrizes, este documento foi traduzido e publicado no Brasil pelo Ministério da Saúde em 2002, recomendando que deva haver uma razão válida para a interferência no processo natural do parto, devendo o mesmo acontecer com o mínimo de intervenções?2.

Estes documentos classificaram as práticas comuns na condução do parto normal em categorias de acordo com sua utilidade, eficácia e risco. 0 uso liberal ou rotineiro da episiotomia, incisão cirúrgica realizada na região perineal da mulher durante o par to vaginal, foi classificado como prática freqüentemente utilizada de modo inadequado, devendo ser realizada somente nos casos em que houver necessidade, indicada em cerca de $10 \%$ a $15 \%$ dos casos $^{1-2}$.

Uma revisão sistemática de ensaios clínicos randomizados e controlados sobre o uso da episiotomia no parto vaginal, desenvolvida e publicada pela biblioteca Cochrane em 2007, evidenciou que o uso restrito da intervenção confere diversos benefícios, como o menor risco de lesões no períneo posterior, menos necessidade de sutura e menos complicações no processo de cicatrização. 0 estudo levantou apenas uma desvantagem da prática seletiva do procedimento, como o aumento da probabilidade de ocorrer lesões perineais anteriores, geralmente lacerações de primeiro e segundo graus ${ }^{3}$.

No parto vaginal, a ocorrência de lacerações perineais depende de vários fatores, que podem estar relacionados às condições maternas e fetais, ao parto em si e à prática da episiotomia, que pode aumentar a gravidade da laceração. As lacerações espontâneas são classificadas como de primeiro grau quando afetam pele e mucosa; de segundo grau quando se estendem até os músculos perineais; de terceiro grau quando atingem o músculo esfíncter do ânus 4 ; e quarto grau quando a lesão do períneo envolve o conjunto do esfíncter anal e exposição do epitélio anal ${ }^{5}$. No caso da episiotomia, além de pele e da mucosa, são habitualmente seccionados os músculos transversos superficial do períneo e bulbocavernos $0^{4}$.

No Brasil e na América Latina, a recomendação do uso restrito do procedimento é desconsiderada. A episiotomia é realizada em mais de $90 \%$ dos partos vaginais ocorridos na América Latina ${ }^{6}$ e no Brasil, sendo efetuada em 94,2\% das primíparas que tiveram parto vaginal ${ }^{7}$. Isso significa que milhões de mulheres, ao serem atendidas em instituições de saúde para dar à luz, têm sua vulva e vagina "cortadas" " "costuradas", sem qualquer indicação médica ${ }^{6}$.

As justificativas apresentadas pela literatura para a realização da episiotomia são diversas, como a prevenção de laceração perineal, de relaxamento do assoalho pélvico e de traumatismo contra a cabeça fetal, além de acelerar o trabalho de parto ${ }^{8}$. Entretanto, a episiotomia é também um agravante para as lacerações perineais de $3^{\circ}$ e $4^{\circ}$ graus, podendo resultar em incontinência fecal, estreitamento excessivo do intróito vaginal, hematoma, edema, infecção e deiscência da sutura ${ }^{9-10}$.

Segundo a evidência científica, as lacerações perineais de $1^{\circ}$ e $2^{\circ}$ graus apresentam melhores resultados que a episiotomia em termos de perda sanguínea, dor, dispareunia, cicatrização e retomada da função muscular. Portanto, comparadas com a intervenção, as lacerações acarretam menos prejuízo para a mãe $e^{11-12}$. Contudo, na maioria dos partos hospitalares, a episiotomia é praticada como medida para prevenção das lacerações perineais ${ }^{13}$, apesar das evidências científicas.

As condutas arriscadas realizadas desnecessariamente, como a episiotomia e a posterior episiorrafia, são consideradas violações do direito da mulher à sua integridade corporal, e a imposição autoritária e não informada desses procedimentos atenta contra o direito à condição de pessoa ${ }^{14}$. De acordo com a Rede Feminista de Saúde, a efetuação de procedimentos desnecessários é também um exemplo de violação do direito humano de estar livre de tratamentos cruéis, humilhantes e degradantes ${ }^{15}$.

Por isso, a assistência ao parto no Brasil é freqüentemente vista como uma forma de violência contra as mulheres, na qual são despersonalizadas, desumanizadas e submetidas a uma série de intervenções, muitas vezes desnecessárias, como a prática habitual da episiotomia. Porém, os preconceitos ainda presentes na formação do profissional de saúde e na organização dos hospitais fazem com que as freqüentes violações dos direitos humanos e direitos reprodutivos conquistados pelas mulheres sejam incorporadas e passem a fazer parte da "rotina normal" da assistência obstétrica ${ }^{15}$.

Fundamentando-se na importância do resgate do papel central da mulher durante o parto e na crescente especulação de hospitais ditos como "humanizados", além do respeito às recomendações da OMS e Ministério da Saúde do Brasil, considera-se importante levantar o número de episiotomias realizadas nas instituiç̧ões hospitalares brasileiras, analisando também a quantidade de lacerações ocorridas durante 0 processo de parturição por via baixa para se avaliar o número de mulheres que sofrem algum tipo de lesão perineal durante este período e, assim, implementar a assistência à saúde da mulher no ciclo gravídico-puerperal.

\section{OBJ ETIVOS}

- Descrever o perfil das mulheres que deram à luz por via vaginal, atendidas em um hospital do interior de São Paulo;

- Levantar a freqüência de lesões perineais ocorridas durante o parto vaginal. 


\section{METODOLOGIA}

Trata-se de um estudo descritivo, com abordagem quantitativa, a respeito da freqüência de lesões perineais no parto vaginal, ocorridas em mulheres que deram à luz por via baixa, em um hospital filantrópico localizado no interior de São Paulo, no período de julho a dezembro de 2006. Nesta instituição, somente no ano de 2006, foram realizados 1.884 partos, sendo que, apenas no mês de julho do referido ano, 0 hospital assistiu 152 partos, dos quais $90(59,21 \%)$ foram cesarianas, $59(38,81 \%)$ partos vaginais e $3(1,97 \%)$ com aplicação de fórceps.

As gestantes que são admitidas no hospital em trabalho de parto são assistidas na sala de pré-parto até o início do período expulsivo, quando a mesma é encaminhada à sala de parto, onde acontece o nascimento. Após o parto, as mulheres permanecem internadas por 24 horas para avaliação pós-parto. Ressalta-se que, no local de estudo, não existe a participação de enfermeiras obstétricas na assistência ao parto e o hospital não classifica sua assistência como humanizada.

A pesquisa foi realizada por meio de análise de prontuários das mulheres que deram à luz por via baixa no referido hospital, no período de julho a dezembro de 2006. Estes documentos

\section{RESULTADOS E DISCUSSÃO}

Foram registrados, no período de julho a dezembro de 2006, 333 partos via baixa na instituição. Não foram localizados 54 prontuários, pois alguns não se encontravam no Serviço de Arquivo Médico e Estático, outros não haviam sido armazenados na sequêencia correta, o que dificultou sua localização.

Assim, foram analisados 279 prontuários de mulheres que tiveram partos vaginais na referida instituição hospitalar. Destes partos, quase a totalidade, 276 (98,92\%), foram acompanhados por médicos obstetras do hospital, os demais encontravam-se armazenados no Serviço de Arquivo Médico e Estático (SAME) da instituição. 0 tamanho da amostra foi determinado de acordo com a representatividade do número de partos ocorridos no período de um ano. Foram excluídas do estudo as mulheres que foram submetidas ao parto com aplicação de fórceps e as cesarianas.

Os dados foram coletados entre os meses de setembro e outubro de 2007, pelas próprias pesquisadoras, com uso de um formulário contendo a identificação da parturiente e informações referentes à história obstétrica, ao parto atual, ao recém-nascido e às condições do períneo após o parto.

As informações obtidas foram armazenadas no aplicativo Excel e analisadas de maneira descritiva, calculando-se as freqüências absolutas e relativas para as variáveis qualitativas e com medidas de tendência central para as variáveis quantitativas.

Para o desenvolvimento do estudo, foram seguidas as diretrizes e normas regulamentadoras de pesquisas que envolvem seres humanos, aprovadas pelo Conselho Nacional de Saúde - Resolução 196/96 16 . 0 projeto de pesquisa foi submetido à análise do Comitê de Ética em Pesquisa da Universidade Paulista de Campinas (UNIP) e aprovado sob protocolo número 241/07.

foram assistidos por outros profissionais da saúde em virtude da iminência do parto.

Com relação ao perfil das mulheres, quando analisada a idade das parturientes (Tabela 1), observou-se que a amplitude da idade das mulheres atendidas na instituição durante 0 período de estudo foi de 12 a 46 anos, destas 33,33\% estavam na faixa etária dos 17 aos 21 anos e 26,88\% tinham entre 22 e 26 anos. A média de idade encontrada foi de 24 anos, com moda de 19 anos e mediana de 24 anos. Observa-se, por meio da análise das medidas de tendência central, que a maioria das mulheres pariu na idade fértil.

\section{Tabela 1 - Distribuição segundo a idade das parturientes atendidas em um hospital no período de julho a dezembro de 2006.}

\begin{tabular}{|c|c|c|c|}
\hline Idode (onos) & $\begin{array}{c}\mathrm{N}^{\circ} \text { Absoluto } \\
\text { (N) }\end{array}$ & $\begin{array}{c}N^{*} \text { Relativo } \\
{[\%]}\end{array}$ & $\begin{array}{c}\text { Porcentagem Acumulada } \\
\qquad \%\end{array}$ \\
\hline $12-16$ & 19 & 6,81 & 6,81 \\
\hline $17-21$ & 93 & 33,33 & 40,14 \\
\hline $22-26$ & 75 & 26,88 & 67,03 \\
\hline $27-31$ & 50 & 17,92 & 84,95 \\
\hline $32-36$ & 25 & 8,96 & 93,91 \\
\hline $37-41$ & 12 & 4,30 & 98,21 \\
\hline $42-46$ & 5 & 1,79 & 100,00 \\
\hline Total & 279 & 100,00 & \\
\hline
\end{tabular}

Fonte: Hospital Filantrópico Interior de SP, 2007. 
A gestação precoce, ou seja, antes dos 18 anos de idade, é um determinante para que a episiotomia seja realizada. A principal justificativa para a prática dessa intervenção em adolescentes seria prevenção de lesões perineais espontâneas graves. Outra explicação para a adoção rotineira da episiotomia em mulheres jovens seria o fato de a pelve materna não ter atingido o padrão adulto, ou a inexperiência do trabalho de parto ${ }^{8}$. Para as mulheres com idade superior a 35 anos $(7,16 \%)$ e que estejam parindo pela primeira vez, a episiotomia é indicada devido à rigidez de tecidos do períneo, porém as chances de a mulher sofrer uma laceração perineal grave é ainda maior ${ }^{17}$.

Em relação à escolaridade, a maioria das mulheres $(50,18 \%)$ estudou menos de oito anos. A baixa escolaridade dificulta o entendimento da fisiologia do nascimento e reduz a probabilidade de a mulher conhecer seu corpo e as modificações decorrentes de sua gestação, como também os seus direitos durante o ciclo gravídico-puerperal. Assim, acaba sofrendo uma série de intervenções obstétricas, sem ao menos questionar sua realização. A maioria das mulheres pesquisadas $(70,50 \%)$ não possui parceiro fixo ou não possuía uma união formal com seu parceiro no momento do parto. Uma situação conjugal insegura também contribui para uma condição desfavorável à gravidez, sendo apontada como um dos fatores de risco gestacional, pois as mulheres sem companheiro fixo estão mais expostas às adversidades no contexto da vida, como a maior predisposição às doenças sexualmente transmissíveis (DST) ${ }^{18}$.

Outro fator determinante para a realização da episiotomia, segundo a literatura, é a história obstétrica materna. Verificamos no estudo que menos da metade das participantes do estudo $(42,29 \%)$ eram primíparas, ou seja, pariram pela primeira vez, enquanto $57,70 \%$ já tiveram partos anteriores (Tabela 2).

Tabela 2 - Distribuição segundo a gestação das parturientes atendidas em um hospital no período de julho a dezembro de 2006.

\begin{tabular}{ccc}
\hline & $N^{\circ}$ Absoluto & $N^{\circ}$ Relativo \\
Gestaçāo & $\left(\mathrm{N}^{0}\right)$ & $(\%)$ \\
\hline Primípara & 118 & 42,29 \\
Secundipara & 80 & 28,67 \\
Multípara & 81 & 29,03 \\
\hline Total & 279 & 100,00
\end{tabular}

\section{Fonte: Hospital Filantrópico Interior de SP, 2007.}

Sabe-se que a episiotomia está indicada para todas as parturientes primíparas, com o objetivo de prevenir lacerações perineais espontâneas ${ }^{17}$. Desse modo, era esperado no estudo a realização da intervenção em aproximadamente $42,0 \%$ das mulheres, sob a justificativa de que o períneo feminino possui menos elasticidade quando a mulher não tem parto anterior, aumentando a probabilidade de ocorrência de laceração perineal. Assim, a episiotomia deve ser realizada para a prevenção de ruptura espontânea, sendo adotada de forma rotineira em primíparas ${ }^{17}$.

Como no Brasil a prática da episiotomia é rotineira, era esperado que as mulheres com parto vaginal anterior já tivessem sofrido a intervenção anteriormente. Isso poderia ser indício para uma nova realização do procedimento, considerando-se as recomendações de renomados autores, que indicam a episiotomia para mulheres com episiotomia anterior ${ }^{17}$. Assim, seguindo este preceito, a maioria das mulheres que pariram na instituição $(57,70 \%)$ deveria ter seu períneo cortado cirurgicamente durante o parto.

Esta situação é grave e equivocada, pois descaracteriza a assistência humanizada ao trabalho de parto e estimula a prática rotineira da intervenção. Seguindo as recomendações desta literatura, observa-se que todas as mulheres devem ser submetidas ao corte cirúrgico, pois descreve que "a episiotomia, quase sempre, indispensável nas primiparturientes, e nas multiparas nas quais tenha sido anteriormente praticada"17 p${ }^{193}$. Assim, a intervenção é recomendada para todas mulheres que dão à luz via baixa, contrariando a evidência científica.

Conforme a idade gestacional das parturientes, a maioria das gestações $(84,17 \%)$ se estendeu de 37 semanas a 41 semanas de idade gestacional, considerada tempo hábil para o parto pela maturidade fetal. Somente $5,04 \%$ das gestações finalizaram antes de 37 semanas e 10,79\% após 41 semanas de idade gestacional. A episiotomia é indicada para partos prematuros (antes de 37 semanas de idade gestacional), pois protege os fetos prematuros da intensa pressão causada por um períneo excessivamente distendido, facilita 0 desprendimento fetal e diminui a ocorrência de hemorragia intraventricular ${ }^{3}$.Para os partos ocorridos após 41 semanas de idade gestacional, a episiotomia é justificada em casos em que esteja ocorrendo sofrimento fetal ou devido à macrossomia fetal, que, nesse caso, causaria laceraç̧ões espontâneas graves ${ }^{3}$.

A alta taxa de episiotomia realizada na instituição também não se justifica pela presença de complicações durante o parto, 
visto que a maioria dos partos vaginais $(83,70 \%)$ ocorreu sem qualquer tipo de alteração obstétrica.

Verificou-se, assim, que a história obstétrica, a idade gestacional e a presença de complicações obstétricas são fatores que não influenciaram na decisão de realizar a intervenção. Nesse contexto, podemos afirmar que a episiotomia não foi utilizada conforme sua necessidade. Sendo assim, o parto vaginal não evoluiu espontaneamente e foi abreviado pelo procedimento, realizado sem indicação precisa.

Com relação ao peso do recém-nascido, outro fator de indicação da intervenção, a média de peso foi de 3.146 gramas (g), com mediana $3.130 \mathrm{~g}$ e moda $3.240 \mathrm{~g}$. 0 baixo peso dos recém-nascidos (fetos abaixo de 1.500 gramas) constitui um fator para a indicação da episiotomia. A principal explicação para a realização dessa intervenção é proporcionar ao feto uma saída menos traumática e um período expulsivo reduzido. Para os fetos com peso excessivo (acima de 3.800 gramas), a indicação do procedimento é devido ao risco de lesão perineal espontâneo grave, que poderia provocar nas parturientes ${ }^{8}$. evidente no estudo que o baixo peso dos recém-nascidos ou o risco de lacerações graves no períneo, em conseqüência do peso excessivo dos recém-nascidos, também não foram critérios para justificar a realização da episiotomia.

Referente às lacerações perineais, identificou-se que estas não foram relatadas na maioria dos prontuários, pois, dos 279 prontuários, 153 (54,83\%) não descreveram a ocorrência de lacerações perineais. Assim, a coleta de dados ficou comprometida devido à falta de dados e informações sobre esse tipo de lesão.

Entre os 279 prontuários estudados, não foi descrita a presença de lesão perineal em 33 deles, o equivalente a $11,83 \%$ dos prontuários analisados. Dentre os 246 que apresentaram a condição perineal após o parto, observou-se que a maioria das mulheres $(90,24 \%)$ foi submetida à episiotomia (Tabela 3). Ressalta-se que a justificativa para a prática do procedimento não foi apresentada em nenhum prontuário analisado.

\section{Tabela 3 - Distribuição segundo as condições do períneo após o parto das mulheres atendidas em um hospital no período de julho a dezembro de 2006.}

\begin{tabular}{ccc}
\hline & $N^{0}$ Absoluto & $N^{0}$ Relativo \\
Tipo de Lesõo Perineal & $\left(\mathrm{N}^{0}\right)$ & $(\%)$ \\
\hline Episiotomia & 214 & 86,99 \\
Episiotomia e Laceração & 8 & 3,25 \\
Períneo Íntegro & 24 & 9,76 \\
\hline Total & $246^{*}$ & 100,00 \\
\hline
\end{tabular}

Fonte: Hospital Filantrópico Interior de SP, 2007.

\section{*Não Relatado: 33 prontuários.}

As lacerações perineais estavam registradas em apenas oito $(3,25 \%)$ prontuários, mas associadas à presença de episiotomia. Em nenhum prontuário foi encontrada somente a descrição da presença de laceração no períneo, o que fortalece ainda mais a premissa de que a intervenção cirúrgica é realizada habitualmente na instituição.

Segundo as recomendações da OMS, a episiotomia deve ser realizada somente em casos específicos, como sofrimento fetal, progressão insuficiente do parto e ameaça de lesão perineal espontânea grave 1 . Podemos inferir por meio da análise destes resultados que, na instituição em estudo, a intervenção é realizada como parte da rotina da assistência obstétrica, não existindo uma prévia avaliação das condições materno-fetais e o julgamento das vantagens e desvantagens dessa prática. Evidenciam-se o mecanicismo e a medicalização do corpo feminino, tornando-o uma apropriação médica, na qual intervenções desnecessárias, que podem ser arriscadas, são praticadas de forma abusiva.

A violência da imposição de rotinas e as interferências obstétricas sem necessidade perturbam e inibem 0 desencadeamento natural dos mecanismos fisiológicos do processo de parturição, que passam a ser sinônimo de patologia e de intervenção, transformando a experiência do parto em uma vivência de terror, alienação e dor ${ }^{15}$.

Para o feminismo, a humanização do parto refere-se ao respeito e à promoção dos direitos de mulheres e crianças, a uma assistência com base na evidência científica e não na conveniência de instituições ou profissionais ${ }^{14}$. Acredita-se que, havendo a desmedicalização do parto e a adoção de técnicas mais humanizadas na obstetrícia, é possível reduzir as altas taxas de lesões perineais sofridas pelas parturientes, garantindo assim a qualidade e a segurança da assistência prestada como 
também reduzindo a chance da vivência negativa do parto para as mulheres ${ }^{18-19}$.

A manutenção da integridade perineal foi descrita somente em 24 prontuários $(9,76 \%)$, nos quais foi relatado que a mulher não sofreu episiotomia ou laceração espontânea. Considera-se uma parcela muito pequena de mulheres com períneo íntegro, se comparada com a quantidade de mulheres que sofreram episiotomia.

Comparando os dados de diferentes paradigmas assistenciais em obstetrícia, observamos a discrepância da realização de partos operatórios e da episiotomia, como é o caso de um Centro de Parto Normal, localizado na região metropolitana de São Paulo, que presta assistência humanizada à mulher durante o trabalho de parto e parto. Conforme relatório estatístico da instituição, somente no mês de fevereiro de 2007 registraram-se 1.633 partos, dos quais $77,2 \%$ foram partos normais, $19,9 \%$ cesarianas e $0,3 \%$ partos com aplicação de fórceps. Com relação à condição perineal, $37,2 \%$ das mulheres tiveram períneo íntegro, $26,8 \%$ sofreram episiotomia, $26,6 \%$ e 9,0\% tiveram laceração de primeiro e segundo grau, respectivamente, e apenas $0,4 \%$ sofreram laceração de terceiro $\mathrm{grau}^{20}$.

Avaliando o exemplo do Centro de Parto Normal, acreditase que é possível a adequação das instituiç̃oes de saúde e de seus profissionais à prática obstétrica mais individualizada e menos intervencionista. Torna-se urgente repensar a aplicação rotineira de diversas práticas de assistência ao parto, que não trazem benefícios, privam a mulher de ter maior controle sobre seu próprio processo de parturição e são associadas a experiências negativas do parto.

\section{CONCLUSÃO}

A prática da episiotomia não foi justificada conforme as suas indicações reais. A sua realização ocorreu sem qualquer enfoque seletivo, sendo praticada rotineiramente na instituição. Fica evidente que a medicalização do parto está presente na instituição hospitalar. Mesmo com a evolução obstétrica e a evidência científica, a realização de procedimento cirúrgico no períneo ainda é freqüente.

É necessário rever as práticas de atendimento à mulher, considerando as evidências científicas e as condutas individualizadas. Nesse sentido, há necessidade de estimular modelos de atendimento mais humanizados aos médicos e enfermeiros, visando à sensibilização desses profissionais, para que respeitem a singularidade de cada parturiente.

A prática freqüente de condutas obstétricas desnecessárias e arriscadas é considerada uma violação do direito à integridade corporal da mulher e um desrespeito aos seus direitos reprodutivos e sexuais. Acredita-se que a expansão e a prática efetiva da humanização do nascimento e do parto contribuirão de modo mais significativo na preservação da integridade emocional e física da parturiente. Respeitando a individualidade feminina, os direitos humanos das mulheres e a prática da obstetrícia baseada na evidência científica, garantimos um parto mais seguro, de qualidade e com melhores resultados maternos e perinatais.

\section{Referências}

1. Organização Mundial de Saúde -OMS. Assistência ao parto normal: um guia prático. Brasília (DF); 1996.

2. Ministério da Saúde (BR). Parto, aborto e puerpério: assistência humanizada à mulher. $1^{\circ}$ ed. Brasília (DF); 2001.

3. Carroli G, Belizan J. Episiotomy for vaginal birth. (Cochrane Review). In: The Cochrane Library 2007.0xford (USA): Update Software.

4. Scarabotto LB, Riesco MLG. Fatores relacionados ao trauma perineal no parto normal em nulíparas. Rev Enferm USP 2006 maio; 40(3): 389-95.

5. Kettle C, O'Brien PMS. Methods and materials used in perineal repair. RCOG. Guideline 2004; 23: 1-8

6. Tomasso $\mathrm{G}$, et al ?Debemos seguir haciendo la episiotomia em forma rutinaria? Rev Obstet Ginecol, Venezuela, 2002; 62 (2): 155221.

7. Althabe F, Belizan JM, Bergel E. Episiotomy rates in primiparous women in Latin American: hospital based descriptive estudy. BMJ 2002; 324 (7343): 945- 46.

8. Junior EFM, Lima MC, Freire S. Fatores associados à realização seletiva de episiotomia em hospital universitário. Rev Cienc Med 2006 abr; 15 (2): 95-101.

9. Progianti JM, Vargens OMC, Porfírio AB, Lorenzoni DP. A preservação perineal como prática de enfermeiras obstétricas. Esc Anna Nery Rev Enferm 2006 ago; 10 (2): 266-73.

10. Costa SM, Ramos JGL, Britzke E, Stuczinski JV, Dias EC. Episiotomia: 0 que há de verdade neste tradicional procedimento. Femina 2001 maio; 29 (4): 201-4.

11. Beleza ACS, Nakano MAS, Santos CB. Práticas obstétricas: fatores de risco para o trauma perineal no parto. Femina 2004 ago; 32 (7): 605-09.

12. Teixeira CEG, Sperotto FC, Salzano LR, Magalhães JAA. Episiotomia e reparo. Rev HCPA 1988 ago; 8 (2): 20-2.

13. Costa ASC. Proteção perineal e manejo do feto no parto normal: estudo comparativo entre duas técnicas. [dissertação de mestrado]. São Paulo (SP): Escola de Enfermagem/ USP; 2002.

14. Diniz CSC. Entre a técnica e os direitos humanos: possibilidades e limites da humanização da assistência ao parto. [tese de doutorado]. São Paulo (SP): Faculdade de Medicina/USP; 2005.

15. Rede Feminista de Saúde. Dossiê humanização do parto: direitos sexuais e direitos reprodutivos. $3^{\mathrm{a}}$ ed. São Paulo (SP); 2002.

16. Ministério da Saúde (BR). Secretaria de Assistência à Saúde. Conselho Nacional de Saúde. Programa Nacional de Doenças Sexualmente Transmissíveis/AIDS. Brasília (DF); 1997.

17. Rezende J, Montenegro CAB. Obstetrícia fundamental. $9^{a}$. Rio de Janeiro (RJ); 2003.

18. Bezerra MGA, Cardoso MVLML. Fatores culturais que interferem nas experiências das mulheres durante o trabalho de parto e parto. Rev Latino-am Enfermagem 2006 jun; 14 (3): 414-21.

19. Davim MB, Eders $B C$, Reis MN. Estudo retrospectivo quanto à prática da episiotomia e a ocorrência de lacerações perineais em uma maternidade-escola. Nurs 2003; 6 (62): 38-42.

Amparo Maternal. [homepage na Internet]. [citado 28 jul 2007]. Disponível em: www.amparomaternal.org 